\title{
NON-GAUSSIAN WICK CALCULUS BASED ON HYPERCOMPLEX SYSTEMS
}

\author{
Abd-Allah Hyder ${ }^{1}$, M. Zakarya ${ }^{2} \S$ \\ ${ }^{1}$ Department of Mathematics \\ Faculty of Science \\ Al-Azhar University \\ Naser City, Cairo, EGYPT \\ ${ }^{2}$ Department of Mathematics \\ Faculty of Science \\ Al-Azhar University \\ Assiut 71524, EGYPT
}

\begin{abstract}
In this paper, we developed a non-Gaussian Wick calculus based on the theory of hypecomplex systems $L_{1}(Q, d m(x))$. Using the Delsarte characters $\chi_{n}(x)$, we introduce a $\chi$-Wick product, a $\chi$-Hermite transform on the space of generalized functions $H_{-q}^{\chi}$ and discuss their properties. By means of the usual properties of complex analytic functions, we proved the characterization theorem for $H_{-q}^{\chi}$. Moreover, we setup a framework to study the stochastic partial differential equations driven by $H_{-q}^{\chi}$-processes, and apply this framework to solve the $\chi$-stochastic Poisson equation.
\end{abstract}

AMS Subject Classification: 46FXX, 30G35, 91G80

Key Words: non-Gaussian, Wick product, Hermite transform, white noise, hypercomplex systems

\section{Introduction}

White noise analysis is an important and popular theme which is intensively

Received: $\quad$ April 25, 2016

Revised: July 27, 2016

Published: September 30, 2016

${ }^{\S}$ Correspondence author (c) 2016 Academic Publications, Ltd. url: www.acadpubl.eu 
and extensively studied in many works (see e.g. the books [21], [22] and references therein). These investigations are essentially based on the concept of a Gaussian measure and the associated expansion into Hermite polynomials. Gaussian measures are remarkable objects, but they are not invariant (as a class of measures) with respect to nonlinear transformations. Moreover, in applications to some problems of mathematical physics, we need to use measures which are obtained as very singular perturbations of Gaussian ones (as in quantum field theory) or are constructed by the Gibbs approach (as in statistical physics) [23].

The classical white noise analysis (Gaussian white noise analysis) it is possible to understand as a theory of generalized functions of infinite many variables with pairing between test and generalized functions provided by integration with respect to the Gaussian measure. Recently, some authors as Okb El Bab, Zabel, Ghany and Hyder [10], Ghany [11], Ghany and Hyder [12, 13, 14], Ghany and Zakarya [15, 16, 17] and Ghany and Qurashi [18], studied some important subjects related to Gaussian white noise analysis. Also, Okb El Bab, Zabel and Ghany [26], introduced some studies of harmonic analysis in hypercomplex systems. Furthermore, Okb El Bab, Ghany and Zakarya [27], studied some important subjects related to a construction of non-Gaussian white noise analysis using the theory of hypercomplex systems. It is well known that there exist several approaches to the construction of such theory of generalized functions: the Berezansky-Samoilenko approach [3] and the Hida approach [20]. In the Berezansky-Samoilenko approach, spaces of test and generalized functions are constructed as infinite tensor products of one-dimensional spaces. The Hida approach consists in the construction of some rigging of a Fock space with subsequent application of the Wiener-Itô-Segal isomorphismto the spaces of this rigging. After a number of years it became clear that the Hida approach is more convenient, and in most cases all investigations in white noise analysis and its generalizations are based on this approach.

There exist many works dedicated to white noise analysis development. In recent years, two approaches to the generalization of the Gaussian infinitedimensional analysis (white noise analysis) to non-Gaussian measures have appeared: one of them is based on spectral theory for families of commuting self-adjoint operators $[4,5]$ and the other proceeds from biorthogonal expansions [1]. Moreover, in the one-dimensional model case, the second approach can be extensively generalized if the characters of an hypercomplex system are used, in its construction, instead of exponential functions.

Now, we recall, in brief, the properties and objects of hypercomplex systems, which we need in our paper. For more details see [2]. 
Consider a commutative hypercomplex system $L_{1}(Q, d m(x))$ of functions $f$ with locally compact basis $Q$, nonnegative structure measure $c(A, B, x)(A, B \in$ $\mathcal{B}(Q), x \in Q)$ and multiplicative measure $d m(x)(x \in Q) ; L_{1}(Q, d m(x))$ is assumed to be normal $\left(Q \ni x \mapsto x^{*} \in Q\right.$ is the corresponding involution in the basis) and to have basis unity $e \in Q$. We can introduce generalized translation operators for these hypercomplex systems, namely for each function $f \in C(Q)$ we construct the function $Q \times Q \ni\langle x, y\rangle \mapsto\left(T_{x} f\right)(y) \in \mathbb{C}$ (which is equal to $f(x+y)$ in the case of ordinary translation on $Q=\mathbb{R})$. We impose an additional condition on $L_{1}(Q, d m(x))$ : let the function $\left(T_{x} f\right)(y)$ be separately continuous. Using the structure measure, the convolution of functions on $Q$ is defined for general hypecomplex system. But the above mentioned restrictions on $L_{1}(Q, d m(x))$ give the possibility to write this convolution in terms of the operators $T_{x}$, as follows:

$$
(f * g)(x)=\int_{Q} f(y)\left(T_{y^{*}} g\right)(x) d m(y), \quad f, g \in L_{1}(Q, d m(x)) .
$$

The hypecomplex system $L_{1}(Q, d m(x))$ with convolution (1.1) is a commutative normed algebra with involution $L_{1}(Q, d m(x)) \ni f \mapsto f^{*} \in L_{1}(Q, d m(x))$, where $f^{*}(x)=\overline{f\left(x^{*}\right)}(x \in Q)$.

A generalized character of the hypercomplex system is defined as a function $\chi \in C(Q)$ such that

$$
\left(T_{x} \chi\right)(y)=\chi(x) \chi(y), \quad x, y \in Q
$$

The character is said to be Hermitian (ordinary) if $\chi\left(x^{*}\right)=\overline{\chi(x)}$ for $x$ (if it is bounded). The function $\chi(x)=1(x \in Q)$ is always a character; $\chi(e)=1$ for any $\chi$.

This paper is devoted to develop a non-Gaussian Wick calculus based on the theory of hypecomplex systems $L_{1}(Q, d m(x))$. Using the Delsarte characters $\chi_{n}(x)$, we introduce a $\chi$-Wick product and $\chi$-Hermite transform on the space of generalized functions $H_{-q}^{\chi}$ (with the zero space $L_{2}(Q, d m(x))$ ) and discuss their properties. By means of the usual properties of complex analytic functions, we prove the characterization theorem for $H_{-q}^{\chi}$. Moreover, we setup a framework to study the stochastic partial differential equations (SPDEs) driven by $H_{-q^{-}}^{\chi}$ processes, and apply this framework to solve the $\chi$-stochastic Poisson equation.

This paper is organized as follows: In Section 2, we recall the rigging of the space $L_{2}(Q, d \rho(x))$ using the Delsarte characters. In Section 3, we introduce the $\chi$-Wick product, the $\chi$-Hermite transform and proved the characterization theorem for the space of generalized functions $H_{-q}^{\chi}$. In Section 4, we give a framework to study the SPDEs driven by $H_{-q}^{\chi}$-processes. In Section 5, we 
apply the framework presented in Section 4 to solve the $\chi$-stochastic Poisson equation.

\section{A rigging of $L_{2}(Q, d \rho(x))$ Using Delsarte Characters}

In this section, we consider a subclass of the above hypercomplex systems for which the set of generalized characters is in one-to-one correspondence with the complex plane: $\chi \longleftrightarrow \lambda \in \mathbb{C}$; denote this character by $\chi(x, \lambda)$. We assume that $\chi(x, 0)=1(x \in Q)$, i.e., the unit character corresponds to $\lambda=0$.

The function $Q \times \mathbb{C} \ni\langle x, \lambda\rangle \mapsto \chi(x, \lambda) \in \mathbb{C}$ is assumed to be continuous, and let the function $\mathbb{C} \ni \lambda \mapsto \chi(x, \lambda) \in \mathbb{C}$ be entire for each $x \in Q$. Thus, for each $x \in Q$ the following expansion holds:

$$
\chi(x, \lambda)=\sum_{n=0}^{\infty} \frac{\lambda^{n}}{n !} \chi_{n}(x), \quad \lambda \in \mathbb{C},
$$

where $\chi_{n} \in C(Q)$ are some coefficients called the Delsarte characters [6, 7]. It is possible, of course, to give a direct definition of the Delsarte characters if we will rewrite equality (2.1) in the terms of $\chi_{n}$.

Let $\rho$ be a fixed Bore1 probability measure on $Q$ i.e., $(\rho(Q)=1)$, positive on open sets, which is not connected directly with the multiplicative measure $m$. We suppose that $\chi_{n}$ 's belong to $L_{2}(Q, d \rho(x))$, for any $n$ and satisfy the estimate

$$
\exists C>0:\left\|\chi_{n}\right\|_{L_{2}(Q, d \rho(x))}=\left(\int_{Q}\left|\chi_{n}(x)\right|^{2} d \rho(x)\right)^{\frac{1}{2}} \leq C^{n} n !, \quad n \in \mathbb{N}
$$

(explaining that always $\left.\left\|\chi_{0}\right\|_{L_{2}(Q, d \rho(x))}=1\right)$, are linearly independent, and the system $\left(\chi_{n}\right)_{n=0}^{\infty}$ is total in $L_{2}(Q, d \rho(x))$.

The following theorem gives the expansions of functions on $Q$ in the Delsarte characters and introduces the corresponding spaces of test and generalized functions.

Theorem 2.1. There exists a quasinuclear rigging:

$$
H_{-q}^{\chi} \supseteq H_{0} \supseteq H_{q}^{\chi}
$$

where $H_{0}=L_{2}(Q, d \rho(x))$, for all $q \in \mathbb{N}$ :

$$
H_{q}^{\chi}=\left\{\varphi=\sum_{n=0}^{\infty} \varphi_{n} \chi_{n} \in H_{0}:\|\varphi\|_{H_{q}^{\chi}}^{2}=\sum_{n=0}^{\infty}\left|\varphi_{n}\right|^{2}(n !)^{2} K^{q n}<\infty\right\},
$$


and

$$
H_{-q}^{\chi}=\left\{\xi=\sum_{n=0}^{\infty} \xi_{n} q_{n}^{\chi}:\|\xi\|_{H_{-q}^{\chi}}^{2}=\sum_{n=0}^{\infty}\left|\xi_{n}\right|^{2} K^{-q n}<\infty \text { for some } q \in \mathbb{N}\right\} .
$$

Here, the system $\left(\chi_{n}, q_{n}^{\chi}\right)_{n=0}^{\infty}$, where $q_{n}^{\chi}=\mathbf{I}^{-1} \chi_{n} \in H_{-q}^{\chi}\left(\mathbf{I}: H_{-q}^{\chi} \rightarrow H_{q}^{\chi}\right.$ is the canonical isometry), is a biorthogonal basis of the space $H_{0}$, and $K$ is a fixed sufficiently large number. Moreover, the dual action is given by

$$
(\xi, \varphi)_{H_{0}}=\sum_{n=0}^{\infty} \xi_{n} \overline{\varphi_{n}}(n !)^{2} K^{q n}, \quad \varphi \in H_{q}^{\chi}, \quad \xi \in H_{-q}^{\chi} .
$$

Proof. The proof can be found in [7].

\section{3. $\chi$-Wick Product, $\chi$-Hermite Transform and Characterization Theorem for $H_{-q}^{\chi}$}

The Wick product was first introduced by Wick [29] and used as a tool to renormalize certain infinite quantities in quantum field theory. Later on, the Wick product was considered, in a stochastic setting, by Hida and Ikeda [19]. In [9], Dobroshin and Minlos were comprehensively treated this subject both in mathematical physics and probability theory. Currently, the Wick product provides a useful concept for various applications, for example, it is important in the study of stochastic ordinary and partial differential equations (see, e.g., $[24])$.

In this section, we define a new Wick product, called $\chi$-Wick product, on the space $H_{-q}^{\chi}$ with respect to an arbitrary Borel probability measure $\rho$. Then, we give the definition of the $\chi$-Hermite transform and apply it to establish a characterization theorem for the space $H_{-q}^{\chi}$.

Definition 3.1. Let $\xi=\sum_{m=0}^{\infty} \xi_{m} q_{m}^{\chi}, \eta=\sum_{n=0}^{\infty} \eta_{n} q_{n}^{\chi} \in H_{-q}^{\chi}$ with $\xi_{m}, \eta_{n}$ $\in \mathbb{C}$. The $\chi$-Wick product of $\xi, \eta$, denoted by $\xi \diamond_{\chi} \eta$, is defined by the formula

$$
\xi \diamond_{\chi} \eta=\sum_{m, n=0}^{\infty} \xi_{m} \eta_{n} q_{m+n}^{\chi}
$$

It is important to show that the spaces $H_{-q}^{\chi}, H_{q}^{\chi}$ are closed under $\chi$-Wick product.

Lemma 3.1. If $\xi, \eta \in H_{-q}^{\chi}$ and $\varphi, \psi \in H_{q}^{\chi}$, we have 
(i) $\xi \diamond_{\chi} \eta \in H_{-q}^{\chi}$,

(ii) $\varphi \diamond_{\chi} \psi \in H_{q}^{\chi}$.

Proof. If $\xi=\sum_{m=0}^{\infty} \xi_{m} q_{m}^{\chi}, \eta=\sum_{n=0}^{\infty} \eta_{n} q_{n}^{\chi} \in H_{-q}^{\chi}$, then for some $q_{1} \in \mathbb{N}$ we have

$$
\sum_{m=0}^{\infty}\left|\xi_{m}\right|^{2} K^{-q_{1} m}<\infty \text { and } \sum_{n=0}^{\infty}\left|\eta_{n}\right|^{2} K^{-q_{1} n}<\infty
$$

We note that

$$
\xi \diamond_{\chi} \eta=\sum_{m, n=0}^{\infty} \xi_{m} \eta_{n} q_{m+n}^{\chi}=\sum_{l=0}^{\infty}\left(\sum_{m+n=l}^{\infty} \xi_{m} \eta_{n}\right) q_{l}^{\chi}=\sum_{l=0}^{\infty} \zeta_{l} q_{l}^{\chi}
$$

where $\zeta_{l}=\sum_{m+n=l}^{\infty} \xi_{m} \eta_{n}$. With $q=q_{1}+p$ we have

$$
\begin{aligned}
\sum_{l=0}^{\infty}\left|\zeta_{l}\right|^{2} K^{-q l} & =\sum_{l=0}^{\infty}\left|\sum_{m+n=l}^{\infty} \xi_{m} \eta_{n}\right|^{2} K^{-q_{1} l} K^{-p l} \\
& \leq \sum_{l=0}^{\infty}\left(\sum_{m+n=l}^{\infty}\left|\xi_{m}\right|^{2} K^{-q_{1} m}\right)\left(\sum_{m+n=l}^{\infty}\left|\eta_{n}\right|^{2} K^{-q_{1} n}\right) K^{-p l} \\
& \leq\left(\sum_{l=0}^{\infty} K^{-p l}\right)\left(\sum_{m=0}^{\infty}\left|\xi_{m}\right|^{2} K^{-q_{1} m}\right)\left(\sum_{n=0}^{\infty}\left|\eta_{n}\right|^{2} K^{-q_{1} n}\right) \\
& <\infty,
\end{aligned}
$$

which proves (i). The proof of (ii) is similar.

The following important algebraic properties of the $\chi$-Wick product follow directly from Definition 3.1.

Lemma 3.2. For each $\xi, \eta, \zeta \in H_{-q}^{\chi}$, we get

(i) $\xi \diamond_{\chi} \eta=\eta \diamond_{\chi} \xi$ (Commutative law),

(ii) $\xi \diamond_{\chi}\left(\eta \diamond_{\chi} \zeta\right)=\left(\xi \diamond_{\chi} \eta\right) \diamond_{\chi} \zeta($ Associative law),

(iii) $\xi \diamond_{\chi}(\eta+\zeta)=\xi \diamond_{\chi} \eta+\xi \diamond_{\chi} \zeta$ (Distributive law). 
Remark. According to Lemmas 3.1 and 3.2, we can conclude that the spaces $H_{-q}^{\chi}$ and $H_{q}^{\chi}$ form topological algebras with respect to the $\chi$-Wick product.

As shown in Lemmas 3.1 and 3.2, the $\chi$-Wick product satisfies all the ordinary algebraic rules for multiplication. Therefore, one can carry out calculations in much the same way as with usual products. But, there are some problems when limit operations are involved. To treat these situations it is convenient to apply a transformation, called the $\chi$-Hermite transform, which converts $\chi$-Wick products into ordinary (complex) products and convergence in $H_{-q}^{\chi}$ into bounded, pointwise convergence in a certain neighborhood of 0 in $\mathbb{C}$. The original Hermite transform, which first appeared in Lindstrøm et al. [25], has been applied by the authors in many different connections. Now, we give the definition of the $\chi$-Hermite transform and discuss its basic properties.

Definition 3.2. Let $\xi=\sum_{n=0}^{\infty} \xi_{n} q_{n}^{\chi} \in H_{-q}^{\chi}$ with $\xi_{n} \in \mathbb{C}$. Then, the $\chi$-Hermite transform of $\xi$, denoted by $\mathcal{H}_{\chi} \xi$, is defined by

$$
\mathcal{H}_{\chi} \xi(z)=\sum_{n=0}^{\infty} \xi_{n} z^{n} \in \mathbb{C} \quad \text { (when convergent) }
$$

In the following, we define for $0<M, q<\infty$ the neighborhoods $\mathbb{O}_{q}(M)$ of zero in $\mathbb{C}$ by

$$
\mathbb{O}_{q}(M)=\left\{z \in \mathbb{C}: \sum_{n=0}^{\infty}\left|z^{n}\right|^{2} K^{q n}<M^{2}\right\} .
$$

It is easy to see that

$$
q \leq p, N \leq M \Rightarrow \mathbb{O}_{p}(N) \subseteq \mathbb{O}_{q}(M)
$$

Note that if $\xi=\sum_{n=0}^{\infty} \xi_{n} q_{n}^{\chi} \in H_{-q}^{\chi}, z \in \mathbb{O}_{q}(M)$ for some $0<M, q<\infty$, we have the estimate

$$
\begin{aligned}
\sum_{n=0}^{\infty}\left|\xi_{n}\right|\left|z^{n}\right| & =\sum_{n=0}^{\infty}\left|\xi_{n}\right|\left|z^{n}\right| K^{-\frac{q n}{2}} K^{\frac{q n}{2}} \\
& \leq\left(\sum_{n=0}^{\infty}\left|\xi_{n}\right|^{2} K^{-q n}\right)^{\frac{1}{2}}\left(\sum_{n=0}^{\infty}\left|z^{n}\right|^{2} K^{q n}\right)^{\frac{1}{2}} \\
& <M\left(\sum_{n=0}^{\infty}\left|\xi_{n}\right|^{2} K^{-q n}\right)^{\frac{1}{2}}
\end{aligned}
$$




$$
<\infty
$$

The conclusion above can be stated as follows:

Proposition 3.1. If $\xi \in H_{-q}^{\chi}$, then $\mathcal{H}_{\chi} \xi$ converges for all $z \in \mathbb{O}_{q}(M)$ for all $q, M<\infty$.

A useful property of the $\chi$-Hermite transform is that it converts the $\chi$-Wick product into ordinary (complex) product.

Proposition 3.2. If $\xi, \eta \in H_{-q}^{\chi}$, then

$$
\mathcal{H}_{\chi}\left(\xi \diamond_{\chi} \eta\right)(z)=\mathcal{H}_{\chi} \xi(z) \cdot \mathcal{H}_{\chi} \eta(z)
$$

for all z such that $\mathcal{H}_{\chi} \xi$ and $\mathcal{H}_{\chi} \eta$ exist.

Proof. The proof is an immediate consequence of Definitions 3.1 and 3.2.

Let $\xi=\sum_{n=0}^{\infty} \xi_{n} q_{n}^{\chi} \in H_{-q}^{\chi}$, with $\xi_{n} \in \mathbb{R}$. Then, the number $\xi_{0}=\mathcal{H}_{\chi} \xi(0) \in$ $\mathbb{R}$ is called the generalized expectation of $\xi$ and is denoted by $\mathbb{E}(\xi)$. Suppose that $V \ni z \mapsto f(z) \in \mathbb{C}$ is an analytic function, where $V$ is a neighborhood of $\mathbb{E}(\xi)$. Assume that the Taylor series of $f$ around $\mathbb{E}(\xi)$ has coefficients in $\mathbb{R}$. Then, the $\chi$-Wick version $f^{\diamond} \chi$ of $f$ is defined by

$$
H_{-q}^{\chi} \ni \xi \mapsto f^{\diamond} \chi(\xi)=\mathcal{H}^{-1}\left(f \circ \mathcal{H}_{\chi}(\xi)\right) \in H_{-q}^{\chi} .
$$

Example 3.1. If the function $f: \mathbb{C} \rightarrow \mathbb{C}$ is entire, then $f^{\diamond} \chi$ is defined for all $\xi \in H_{-q}^{\chi}$. For example, the $\chi$-Wick exponential is defined by

$$
\exp ^{\diamond} \chi(\xi)=\sum_{j=0}^{\infty} \frac{1}{j !} \xi^{\diamond} \chi^{n}
$$

Using $\chi$-Hermite transform, we see that $\chi$-Wick exponential has the same algebraic properties as the usual exponential. For instance,

$$
\exp ^{\diamond} \chi(\xi+\eta)=\exp ^{\diamond} \chi(\xi) \diamond_{\chi} \exp ^{\diamond} \chi(\eta), \xi, \eta \in H_{-q}^{\chi}
$$

From Proposition 3.1, we can deduce that $\chi$-Hermite transform of any $\xi \in H_{-q}^{\chi}$ is a complex-valued analytic function on $\mathbb{O}_{q}(M)$ for all $q, M<\infty$. Moreover, the converse of this deduction is true, i.e., every complex-valued analytic function on $\mathbb{O}_{q}(M)$ (for some $q, M<\infty$ ) is the $\chi$-Hermite transform of some element in $H_{-q}^{\chi}$. To prove this, we need the following two auxiliary results. 
Lemma 3.3. Let $f(z)=\sum_{n=0}^{\infty} \eta_{n} z^{n}$ be an analytic function in $z \in \mathbb{C}$ such that there exists $M<\infty, C>0$ and $\delta>0$ such that $|f(z)| \leq M$ when $z \in \mathbb{O}:=\left\{z \in \mathbb{C}: C|z| \leq \delta^{2}\right\}$. Then $\left|\eta_{n} z^{n}\right| \leq M$ for all $z \in \mathbb{O}$ and $n \in \mathbb{N}$.

Proof. See [24], Lemma 2.6.10.

Proposition 3.3. Let $f(z)=\sum_{n=0}^{\infty} \eta_{n} z^{n}, \eta_{n} \in \mathbb{C}$ be a formal power series in $z \in \mathbb{C}$. Suppose there exist $q, M<\infty$ and $\delta>0$ such that $f(z)$ is convergent for $z \in \mathbb{O}_{q}(\delta)$ and $|f(z)| \leq M$ for all $z \in \mathbb{O}_{q}(\delta)$. Then

$$
\sum_{n=0}^{\infty}\left|\eta_{n} z^{n}\right| \leq M A(q) \quad \text { for all } z \in \mathbb{O}_{3 q}(\delta)
$$

where

$$
A(q):=\sum_{n=0}^{\infty} K^{-q n}<\infty \quad(\text { Note that } K>1)
$$

Proof. It is evident that $z \in \mathbb{O}_{3 q}(\delta)$ implies $K^{q} z \in \mathbb{O}_{q}(\delta)$. According to Lemma 3.3, we get

$$
\begin{aligned}
\sum_{n=0}^{\infty}\left|\eta_{n} z^{n}\right| & \leq\left(\sum_{n=0}^{\infty}\left|\eta_{n}\right|^{2}\left|z^{n}\right|^{2} K^{q n}\right)^{\frac{1}{2}}\left(\sum_{n=0}^{\infty} K^{-q n}\right)^{\frac{1}{2}} \\
& =\left(\sum_{n=0}^{\infty}\left|\eta_{n}\right|^{2}\left|\left(K^{q} z\right)^{n}\right|^{2} K^{-q n}\right)^{\frac{1}{2}}\left(\sum_{n=0}^{\infty} K^{-q n}\right)^{\frac{1}{2}} \\
& \leq M \sum_{n=0}^{\infty} K^{-q n}
\end{aligned}
$$

Theorem 3.1. (Characterization Theorem for $H_{-q}^{\chi}$ ) If $\xi=\sum_{n=0}^{\infty} \xi_{n} q_{n}^{\chi} \in$ $H_{-q}^{\chi}$, where $\xi_{n} \in \mathbb{C}$, then there exist $q<\infty$ and $R_{q}<\infty$ such that

$$
\left|\mathcal{H}_{\chi} \xi(z)\right| \leq R_{q}\left(\sum_{n=0}^{\infty}\left|z^{n}\right|^{2} K^{q n}\right)^{\frac{1}{2}} \quad \forall z \in \mathbb{C} .
$$

In particular, $\mathcal{H}_{\chi} \xi$ is a bounded analytic function on $\mathbb{O}_{q}(M)$ for all $M<\infty$. Conversely, suppose $f(z)=\sum_{n=0}^{\infty} \eta_{n} z^{n}$ is a given analytic power series of $z \in \mathbb{C}$ with $\eta_{n} \in \mathbb{C}$ such that there exist $q<\infty$ and $\delta>0$, such that $f(z)$ is absolutely convergent when $z \in \mathbb{O}_{q}(\delta)$ and

$$
\sup _{z \in \mathbb{O}_{q}(\delta)}|f(z)|<\infty
$$


Then, there exists a unique $\eta \in H_{-q}^{\chi}$ such that $\mathcal{H}_{\chi} \eta=f$, namely

$$
\eta=\sum_{n=0}^{\infty} \eta_{n} q_{n}^{\chi}
$$

Proof. For each $z \in \mathbb{C}$, we have

$$
\left|\mathcal{H}_{\chi} \xi(z)\right| \leq \sum_{n=0}^{\infty}\left|\xi_{n}\right|\left|z^{n}\right| \leq\left(\sum_{n=0}^{\infty}\left|\xi_{n}\right|^{2} K^{-q n}\right)^{\frac{1}{2}}\left(\sum_{n=0}^{\infty}\left|z^{n}\right|^{2} K^{q n}\right)^{\frac{1}{2}}
$$

Since $\xi \in H_{-q}^{\chi}$, we see that $R_{q}^{2}:=\sum_{n=0}^{\infty}\left|\xi_{n}\right|^{2} K^{-q n}<\infty$ for all $q<\infty$.

Conversely, Since $K>1$, then $K^{-r} \in \mathbb{O}_{r}(\delta)$ for all $r<\infty$ and for some $\delta<\infty$. By virtue of Proposition 3.3, we have

$$
\sum_{n=0}^{\infty}\left|\eta_{n}\right|\left|z^{n}\right| \leq M A(q) \quad \text { for all } z \in \mathbb{O}_{3 q}(\delta)
$$

Hence, for $r \geq 3 q$ and $z \in \mathbb{O}_{3 q}(\delta)$, we get

$$
\sum_{n=0}^{\infty}\left|\eta_{n}\right|^{2} K^{-r n} \leq C \sum_{n=0}^{\infty}\left|\eta_{n}\right| K^{-r n} \leq C \sum_{n=0}^{\infty}\left|\eta_{n}\right|\left|z^{n}\right| \leq C M A(q)<\infty .
$$

where $C:=\sup \left\{\left|\eta_{n}\right|: n \in \mathbb{N}\right\}$, and hence $\eta:=\sum_{n=0}^{\infty} \eta_{n} q_{n}^{\chi} \in H_{-q}^{\chi}$, as claimed.

\section{Framework for $\chi$-SPDEs}

In the Gaussian case, if the objects of a differential equation are regarded as $(\mathcal{S})_{-1}$-valued $\left((\mathcal{S})_{-1}\right.$ is the Kondratiev space of stochastic distributions constructed upon Gaussian measure), we often obtain a more realistic mathematical model of the situation. This model called a Wick-type stochastic differential equation (see [24] for more details). Analogously, we can introduce a non-Gaussian Wick-type stochastic model by the replacement of $(\mathcal{S})_{-1}$ on $H_{-q}^{\chi}$ and the Wick product associated with the Gaussian measure on the $\chi$-Wick product.

One of the many useful properties of $H_{-q}^{\chi}$ it that it contains the $\chi$-white noise, which is defined by the formal expansion

$$
W_{\chi}(t, x)=\sum_{n=0}^{\infty} \chi_{n}(t, x) q_{n}^{\chi}, \quad t \in \mathbb{R}, x \in Q
$$


Obviously, for each $t \in \mathbb{R}$, we have $W_{\chi}(t) \in H_{-q}^{\chi}$. Indeed, by virtue of estimate (2.2), we have $\left|\chi_{n}(t)\right|^{2} \leq C$ for all $n \in \mathbb{N}, t \in \mathbb{R}$ for a constant $C$. Since $K>1$, then for $t \in \mathbb{R}$ we get

$$
\sum_{n=0}^{\infty}\left|\chi_{n}(t)\right|^{2} K^{-q n} \leq C \sum_{n=0}^{\infty} K^{-q n}<\infty
$$

By comparing the expansion (4.3) for $\chi$-white noise with the expansion

$$
B_{\chi}(t, x)=\sum_{n=0}^{\infty} \int_{0}^{t} \chi_{n}(\tau, x) d \tau q_{n}^{\chi}, \quad t \in \mathbb{R}, x \in Q .
$$

for $\chi$-Brownian motion, we see that

$$
W_{\chi}(t)=\frac{d}{d t} B_{\chi}(t) \quad \text { in } \quad H_{-q}^{\chi}
$$

Therefore, one advantage of working in the general space $H_{-q}^{\chi}$ of stochastic distributions is that it contains the solutions of many non-Gaussian stochastic differential equations, both ordinary and partial and in arbitrary dimension. Moreover, if the objects of such equations are regarded as $H_{-q}^{\chi}$-valued, then differentiation can be interpreted in the usual strong sense in $H_{-q}^{\chi}$.

In what follows, by $\chi$-stochastic generalized process or $H_{-q}^{\chi}$-process we mean a measurable function $\mathbb{R}^{n} \ni\left(t_{1}, t_{2}, \ldots, t_{n}\right) \mapsto u\left(t_{1}, t_{2}, \ldots, t_{n}\right) \in H_{-q}^{\chi}$. Also, the process $u$ is called continuous, differentiable, $C^{1}, C^{k}$, etc., if the $H_{-q}^{\chi}$-valued function $u$ has these properties, respectively. In order to investigate the $\chi$ SPDEs, we will give the following definitions and results.

Definition 4.1. The partial derivative $\frac{\partial u}{\partial t_{k}}$ of an $H_{-q}^{\chi}$-process $u$ is defined by

$$
\frac{\partial u}{\partial t_{k}}\left(t_{1}, \ldots, t_{n}\right)=\lim _{\Delta t_{k} \rightarrow 0} \frac{u\left(t_{1}, \ldots, t_{k}+\Delta t_{k}, \ldots, t_{n}\right)-u\left(t_{1}, \ldots, t_{k}, \ldots, t_{n}\right)}{\Delta t_{k}}
$$

provided the limit exists in $H_{-q}^{\chi}$.

In view of $\chi$ - Hermite transform, the limit in equation (4.5) exists if and only if there exists an element $\zeta \in H_{-q}^{\chi}$ such that

$$
\frac{1}{\Delta t_{k}}\left(\mathcal{H}_{\chi} u\left(t_{1}, \ldots, t_{k}+\Delta t_{k}, \ldots, t_{n}, z\right)-\mathcal{H}_{\chi} u\left(t_{1}, \ldots, t_{k}, \ldots, t_{n}, z\right)\right) \rightarrow \mathcal{H}_{\chi} \zeta(z)
$$


pointwise boundedly (or uniformly) in $\mathbb{O}_{q}(M)$ for some $0<M, q<\infty$. Hence, $\zeta$ is the partial derivative of $u$, and is denoted by $\frac{\partial u}{\partial t_{k}}$.

When we apply the $\chi$-Hermite transform to solve the $\chi$-stochastic differential equations the following observation is important.

For simplicity of notation, choose $n=1$ and consider a differentiable $H_{-q}^{\chi}$-process $X(t, x)$. The statement that

$$
\frac{d X(t, x)}{d t}=F(t, x) \text { in } H_{-q}^{\chi}
$$

is then equivalent to saying that

$$
\lim _{\Delta t \rightarrow 0} \frac{1}{\Delta t}\left(\mathcal{H}_{\chi} X(t+\Delta t, z)-\mathcal{H}_{\chi} X(t, z)\right)=\mathcal{H}_{\chi} F(t, z)
$$

pointwise boundedly for $z \in \mathbb{O}_{q}(M)$ for some $0<M, q<\infty$. For this it is clearly necessary that

$$
\frac{d}{d t}\left(\mathcal{H}_{\chi} X(t, x)\right)=\mathcal{H}_{\chi} F(t, x) \text { for each } z \in \mathbb{O}_{q}(M),
$$

but apparently not sufficient, because we also need that the pointwise convergence is bounded for $z \in \mathbb{O}_{q}(M)$. The following result is sufficient for our purposes.

Lemma 4.1 Suppose $X(t, x)$ and $F(t, x)$ are $H_{-q}^{\chi}$-processes such that

(i) $\frac{d}{d t}\left(\mathcal{H}_{\chi} X(t, x)\right)=\mathcal{H}_{\chi} F(t, x)$, for each $t \in(a, b), z \in \mathbb{O}_{q}(M)$,

(ii) $\mathcal{H}_{\chi} F(t, z)$ is a bounded function of $(t, z) \in(a, b) \times \mathbb{O}_{q}(M)$, continuous in $t \in(a, b)$ for each $z \in \mathbb{O}_{q}(M)$.

Then $X(t, x)$ is a differentiable $H_{-q}^{\chi}$-process and

$$
\frac{d X(t, x)}{d t}=F(t, x) \text { for all } t \in(a, b)
$$

Proof. By the mean value theorem, we have

$$
\frac{1}{\Delta t}\left(\mathcal{H}_{\chi} X(t+\Delta t, z)-\mathcal{H}_{\chi} X(t, z)\right)=\mathcal{H}_{\chi} F(t+\theta \Delta t, z)
$$

for some $\theta \in[0,1]$ and for each $z \in \mathbb{O}_{q}(M)$. So, if (i) and (ii) hold, then

$$
\frac{1}{\Delta t}\left(\mathcal{H}_{\chi} X(t+\Delta t, z)-\mathcal{H}_{\chi} X(t, z)\right) \rightarrow \mathcal{H}_{\chi} F(t, z) \quad \text { as } \quad \Delta t \rightarrow 0
$$


pointwise boundedly for $z \in \mathbb{O}_{q}(M)$.

Similarly, we can relate the integrability of an $H_{-q}^{\chi}$-process to the integrability of its $\chi$-Hermite transform as follows.

Lemma 4.2. Let $X(t)$ be an $H_{-q}^{\chi}$-process. Suppose there exist $0<M, q<$ $\infty$, such that

(i) $\sup \left\{\mathcal{H}_{\chi} X(t, z), t \in[a, b], z \in \mathbb{O}_{q}(M)\right\}<\infty$,

(ii) $\mathcal{H}_{\chi} X(t, z)$ is a continuous function of $t \in[a, b]$ for each $z \in \mathbb{O}_{q}(M)$.

Then $X(t)$ is strongly integrable and

$$
\mathcal{H}_{\chi}\left[\int_{a}^{b} X(t) d t\right]=\int_{a}^{b} \mathcal{H}_{\chi} X(t) d t .
$$

Proof. As usual, the $H_{-q}^{\chi}$-process $X(t)$ is (strongly) integrable in $H_{-q}^{\chi}$ over the interval $[a, b]$ if

$$
\int_{a}^{b} X(t, x) d t:=\lim _{\Delta t_{k} \rightarrow 0} \sum_{k=0}^{n-1} X\left(t_{k}^{*}, x\right) \Delta t_{k}
$$

exists in $H_{-q}^{\chi}$, for all partitions $a=t_{0}<t_{1}<\ldots<t_{n}=b$ of $[a, b], \Delta t_{k}=$ $t_{k+1}-t_{k}$ and $t_{k}^{*} \in\left[t_{k}, t_{k+1}\right]$ for $k=1, \ldots . n-1$. Taking $\mathcal{H}_{\chi}$-transforms, yields

$$
\begin{aligned}
\mathcal{H}_{\chi}\left[\int_{a}^{b} X(t, x) d t\right] & =\mathcal{H}_{\chi}\left[\lim _{\Delta t_{k} \rightarrow 0} \sum_{k=0}^{n-1} X\left(t_{k}^{*}, x\right) \Delta t_{k}\right] \\
& =\lim _{\Delta t_{k} \rightarrow 0} \sum_{k=0}^{n-1} \mathcal{H}_{\chi} X\left(t_{k}^{*}, z\right) \Delta t_{k} \\
& =\int_{a}^{b} \mathcal{H}_{\chi} X(t) d t,
\end{aligned}
$$

and the lemma is proved. $\quad \square$ In the case of higher order derivatives we have the following result

Lemma 4.3. Suppose there exist an open interval $I$, real numbers $q, M$ and a function $I \times \mathbb{O}_{q}(M) \ni(t, z) \mapsto u(t, z) \in \mathbb{C}$ such that

$$
\frac{\partial^{2} u}{\partial t^{2}}(t, z)=\mathcal{H}_{\chi} F(t, z) \text { for }(t, z) \in I \times \mathbb{O}_{q}(M),
$$


where $F(t) \in H_{-q}^{\chi}$ for all $t \in I$. Suppose $\frac{\partial^{2} u}{\partial t^{2}}(t, z)$ is bounded for $(t, z) \in$ $I \times \mathbb{O}_{q}(M)$ and continuous with respect to $t \in I$ for each $z \in \mathbb{O}_{q}(M)$. Then there exists $U(t) \in H_{-q}^{\chi}$ such that

$$
\frac{\partial^{2} U}{\partial t^{2}}=F(t) \text { in } H_{-q}^{\chi}, \text { for all } t \in I
$$

Proof. By the mean value theorem again, we have

$$
\frac{1}{(\Delta t)^{2}}(u(t+2 \Delta t, z)-2 u(t+\Delta t, z)+u(t, z))=\mathcal{H}_{\chi} F(t+\theta \Delta t, z)
$$

for some $\theta \in[0,1]$, for each $z \in \mathbb{O}_{q}(M)$. So, if (4.16) and the assumptions on $\frac{\partial^{2} u}{\partial t^{2}}$ hold, then

$$
\lim _{\Delta t \rightarrow 0} \frac{u(t+2 \Delta t, z)-2 u(t+\Delta t, z)+u(t, z)}{(\Delta t)^{2}}=\mathcal{H}_{\chi} F(t, z)
$$

pointwise boundedly for $z \in \mathbb{O}_{q}(M)$. According to Theorem 3.1, we can apply the inverse Hermite transform to equation (4.19) and get

$$
\frac{\partial^{2} U}{\partial t^{2}}=F(t) \text { in } H_{-q}^{\chi}, \text { for all } t \in I
$$

where $u(t, z)=\mathcal{H}_{\chi} U(t)(z)$ for all $(t, z) \in I \times \mathbb{O}_{q}(M)$.

More generally, we can apply the argument of Lemma 4.1 repeatedly and get the following result

Theorem 4.1. Suppose $u(t, s, z)$ is a solution (in the usual strong, pointwise sense) of the equation

$$
\mathcal{H}_{\chi} A\left(t, s, \partial_{t}, \nabla_{s}, u, z\right)=0
$$

for $(t, s)$ in some bounded open set $D \subset \mathbb{R}_{+} \times \mathbb{R}^{n}$, and for all $z \in \mathbb{O}_{q}(M)$, for some $q, M$. Moreover, suppose that $u(t, s, z)$ and all its partial derivatives, which are involved in equation (4.21) are (uniformly) bounded for $(t, s, z) \in D \times$ $\mathbb{O}_{q}(M)$, continuous with respect to $(t, s) \in D$ for each $z \in \mathbb{O}_{q}(M)$ and analytic with respect to $z \in \mathbb{O}_{q}(M)$, for all $(t, s) \in D$. Then there exists $U(t, s) \in H_{-q}^{\chi}$ such that $u(t, s, z)=\mathcal{H}_{\chi} U(t, s, z)$ for all $(t, s, z) \in D \times \mathbb{O}_{q}(M)$ and $U(t, s)$ solves in the strong sense in $H_{-q}^{\chi}$ the equation

$$
A^{\diamond}\left(t, s, \partial_{t}, \nabla_{s}, U, x\right)=0 \quad \text { in } H_{-q}^{\chi} .
$$




\section{Application to the $\chi$-Stochastic Poisson Equation}

Let us illustrate how the framework, developed in Sections 3,4, can be applied to solve the $\chi$-stochastic Poisson equation [28]

$$
\begin{cases}\Delta U(s)=-W_{\chi}(s), & s \in D, \\ U(s)=0, & s \in \partial D,\end{cases}
$$

where $\Delta=\sum_{k=1}^{n} \frac{\partial^{2}}{\partial s_{k}^{2}}$ is the Laplace operator in $\mathbb{R}^{n}, D \subset \mathbb{R}^{n}$ is a bounded domain with regular boundary $\partial D$ and where $W_{\chi}(s)=\sum_{n=0}^{\infty} \chi_{n}(s) q_{n}^{\chi}$ is $n$ parameter $\chi$-white noise.

The model (5.1) gives a description of the temperature $U(x)$ in the region $D$ under the assumption that the temperature at the boundary is kept equal to zero and that there is a $\chi$-white noise heat source in $D$.

Note that $\Delta U(s)$ in equation (5.1) is defined in the sense of the topology on $H_{-q}^{\chi}$. Now, we aim at converting the system (5.1) into a deterministic partial differential equation (PDE) with complex coefficients by applying the $\chi$-Hermite transform to both sides of equation (5.1). Then, we try to solve the resulting $\mathrm{PDE}$, and we take the inverse $\chi$-Hermite transform of the solution, if existent, to obtain a solution of the original equation.

Taking $\chi$-Hermite transform of equation (5.1), we get

$$
\begin{cases}\Delta u(s, z)=-\mathcal{H}_{\chi} W_{\chi}(s, z), & s \in D, \\ u(s, z)=0, & s \in \partial D,\end{cases}
$$

where $u(s, z)=\mathcal{H}_{\chi} U(s, z)$ and $\mathcal{H}_{\chi} W_{\chi}(s, z)=\sum_{n=0}^{\infty} \chi_{n}(s) z^{n}$ for $z \in \mathbb{C}$. By comparing the real and imaginary parts of equation (5.2), we see that the usual solution formula holds:

$$
u(s, z)=\int_{\mathbb{R}^{n}} G(s, r) \mathcal{H}_{\chi} W_{\chi}(r, z) d r,
$$

where $G(s, r)$ is the classical Green function of $D$ with $G=0$ outside of $D$ (see, e.g., Chapter 9 in $[28])$. Since $G(s,.) \in L_{1}\left(\mathbb{R}^{n}\right)$ for all $s$, the right-hand side of equation (5.3) exists for all $z \in \mathbb{C}$ and $s \in D$. Hence, $u(s, z)$ is defined for such $s, z$.

Further, we have

$$
|u(s, z)|=\left|\int_{\mathbb{R}^{n}} G(s, r) \sum_{n=0}^{\infty} \chi_{n}(r) z^{n} d r\right|=\left|\sum_{n=0}^{\infty} z^{n} \int_{\mathbb{R}^{n}} G(s, r) \chi_{n}(r) d r\right|
$$




$$
\begin{aligned}
& \leq \sum_{n=0}^{\infty}\left|z^{n}\right| \int_{\mathbb{R}^{n}}|G(s, r)|\left|\chi_{n}(r)\right| d r \leq C \sum_{n=0}^{\infty}\left|z^{n}\right| \\
& \leq C\left(\sum_{n=0}^{\infty}\left|z^{n}\right|^{2} K^{2 n}\right)^{\frac{1}{2}}\left(\sum_{n=0}^{\infty} K^{-2 n}\right)^{\frac{1}{2}} \\
& \leq C M\left(\sum_{n=0}^{\infty} K^{-2 n}\right)^{\frac{1}{2}}<\infty
\end{aligned}
$$

for all $z \in \mathbb{O}_{2}(M), M<\infty$. Moreover, equation (5.3) shows that $u(x, z)$ is analytical in $z$. Thus, we conclude by the characterization theorem (Theorem 3.1) that there exists a function $\bar{D} \ni s \mapsto U(s) \in H_{-q}^{\chi}$ such that $\mathcal{H}_{\chi} U(s, z)=$ $u(s, z)$. It is known from the general theory of deterministic elliptic PDEs (see, e.g., [8]) that for all open and relatively compact $V$ in $D$ there exists a constant $C$ such that

$$
\|u(., z)\|_{C^{2+k}(V)} \leq C\left(\|\Delta u(., z)\|_{C^{k}(V)}+\|u(., z)\|_{C(V)}\right)
$$

for all $z \in \mathbb{C}$. Since $\Delta u=-\mathcal{H}_{\chi} W_{\chi}$ and $u$ are bounded on $D \times \mathbb{O}_{2}(M)$, it follows that $\frac{\partial^{2}}{\partial s_{k}} u(s, z)$ is bounded for such $s, z$. Thus, by Theorem $4.1 U$ is a solution of system (5.1).

We conclude directly from equation (5.3) that $u$ is the $\chi$-Hermite transform of

$$
U(s)=\int_{\mathbb{R}^{n}} G(s, r) W_{\chi}(r) d r=\sum_{n=0}^{\infty} \int_{\mathbb{R}^{n}} G(s, r) \chi_{n}(r) d r q_{n}^{\chi},
$$

So we obtain the following result.

Theorem 5.1. The unique $\chi$-stochastic distribution process $U(s) \in H_{-q}^{\chi}$ solving equation (5.1) is given by

$$
U(s)=\int_{\mathbb{R}^{n}} G(s, r) W_{\chi}(r) d r=\sum_{n=0}^{\infty} \int_{\mathbb{R}^{n}} G(s, r) \chi_{n}(r) d r q_{n}^{\chi} .
$$

We have $U(s) \in H_{-q}^{\chi}$ for all $s \in \bar{D}$.

\section{References}

[1] S. Albeverio, Yu. G. Kondratiev and L. Streit, How to generalize white noise analysis to non-Gaussian measures. Proc. Symp. Dynamics of Complex and Irregular Systems (Bielefeld, 1991); Bielefeld Encount. Math. Phys., VIII, World Sci. Publ.: River Edge, NJ (1993), 120-130. 
[2] S. Albeverio, Yu. L. Daletsky, Yu. G. Kondratiev and L. Streit, Non-gaussian infinite dimensional analysis, J. Funct. Anal. 138 (1996), 311-350.

[3] Yu. M. Berezansky and E. V. Lytvynov, Generalized white noise analysis connected with perturbed field operators, Dopovidy AN: Ukrainy, 1993.

[4] Yu. M. Berezansky, Spectral approach to white noise analysis. Proc. Symp. Dynamics of Complex and Irregular Systems (Bielefeld, 1991); Bielefeld Encount. Math. Phys., VIII, World Sci. Publ., River Edge, NJ (1993) 131-140.

[5] Yu. M. Berezansky, V. O. Livinsky and E. W. Lytvynov, A generalization of Gaussian noise analysis, Methods of Functional Analysis and Topology 1 (1995), 28-55.

[6] Yu. M. Berezansky, A connection between the theory of hypergroups and white noise analysis, Reports on Mathematical Physics 36 (1995), 215-234.

[7] Yu. M. Berezansky, A generalization of white noise analysis by means of theory of hypergroups, Reports on Mathematical Physics 38 (1996), 289-300.

[8] L. Bers, F. John and M. Schechter, Partial differential equations, Wiley: New York (1964).

[9] R. L. Dobrushin and R. L. Minlos, Polynomials in linear random functions, Russian Math. Surveys 32 (1977), 71-127.

[10] H. A. Ghany, A. S. Okb El Bab, A. M. Zabel, and A. Hyder, The fractional coupled KdV equations: exact solutions and white noise functional approach, Chinese Physics B, vol. 22, no. 8 (2013) Article ID 080501.

[11] H. A. Ghany, Exact solutions for stochastic generalized Hirota-Satsuma coupled KdV equations, Chinese Journal of Physics, vol. 49, no. 4 (2011) pp. 926940.

[12] H. A. Ghany and A. Hyder, Local and global well-posedness of stochastic ZakharovKuznetsov equation Journal of Computational Analysis and Applications, 15 (7), 13321343

[13] H. A. Ghany and A. Hyder, Exact solutions for the wicktype stochastic time-fractional KdV equations, Kuwait Journal of Science, vol. 41, no. 1,(2014) pp. 7584.

[14] H. A. Ghany and A. Hyder, Abundant solutions of Wick-type stochastic fractional 2D KdV equations, Chinese Physics B,, vol. 23, no. 6,(2014) Article ID 060503.

[15] H. A. Ghany and M. Zakarya, Generalized Solutions of Wick-type Stochastic KdVBurgers Equations Using Exp-function Method International Review of Physics, (IREPHY) 8 (2),(2014) 38-46.

[16] H. A. Ghany and M. Zakarya, Exact Solutions for Wick-type Stochastic Coupled KdV Equations Global Journal of Science Frontier Research: F Mathematics and Decision Sciences, 14 (2014) 56-72.

[17] H. A. Ghany and M. Zakarya, Exact Traveling Wave Solutions for Wick-Type Stochastic Schamel KdV Equation Physics Research International, (2014) p. 1-9

[18] H. A. Ghany and M. A. Qurashi, Travelling Solitary Wave Solutions for Stochastic Kadomtsev-Petviashvili Equation. Journal of Computational Analysis and Applications, 21 (1) (2015) p. 121-131.

[19] T. Hida and N. Ikeda, Analysis on Hilbert space with reproducing kernel arising from multiple Wiener integral. Proc. Fifth Berkeley Symp.Math. Stat. Probab. II (1965), 117143. Univ. California Press, Berkeley. 
[20] T. Hida, Analysis of Brownian functionals, in Carleton Math. Lecture Notes 13, Carleton Univ.: Ottawa 1975.

[21] T. Hida, Brownian motion, Springer-Verlag: Berlin-Heidelberg-New York, 1980.

[22] T. Hida, H. H. Kuo, J. Potthoff, and L. Streit, White noise: An infinite dimensional calculus, Kluwer: Dordrecht (1993).

[23] H. Holden, T. Lindstroøm, B. Ø ksendal, J. Uboø e, and T. Zhang, The pressure equation for fluid flow in a stochastic medium, Pot. Anal. 4 (1995), 655-674.

[24] H. Holden, B. Øsendal, J. Ubøe and T. Zhang, Stochastic partial differential equations, Springer Science+Business Media, LLC (2010).

[25] T. Lindstrøm, B. Øksendal and J. Ubøe, Stochastic differential equations involving positive noise. In M. Barlow and N. Bingham (editors): Stochastic Analysis. Cambridge University Press: Cambridge, MA (1991), 261-303.

[26] A. S. Okb El Bab, A. M. Zabel, H. A. Ghany, Harmonic analysis in Hypercomplex systems, International Journal of Pure and Applied Mathematics, 80 (2012), 739-750.

[27] A. S. Okb El Bab, Hossam A. Ghany and M. Zakarya, A construction of Non-Gaussian White Noise Analysis using the Theory of Hypercomplex Systems, Global Journal of Science Frontier Research: F Mathematics and Decision Sciences, 16 (2016) 11-25.

[28] B. Øksendal, An introduction to Malliavin calculus with applications to economics. Working paper No 3/96, Norwegian School of Economics and Business Administration, 1996.

[29] G. C. Wick, The evaluation of the collinear matrix, Phys. Rev. 80 (1950), 268-272. 